\title{
Kommission Studium und Lehre
}

\section{Kommentar zu Lindner, M. A. et al. (2021). Ein Plädoyer zur Qualitätssicherung schriftlicher Prüfungen im Psychologiestudium}

\author{
Birgit Spinath ${ }^{1}$, Conny H. Antoni ${ }^{2}$, Christina Bermeitinger ${ }^{3}$, Markus Bühner $^{4}$, Birgit Elsner ${ }^{5}$, \\ Edgar Erdfelder ${ }^{6}$, Thomas Fydrich7, Anne Gärtner ${ }^{8}$, Mario Gollwitzer ${ }^{4}$, Cornelius König ${ }^{9}$, \\ und Brunna Tuschen-Caffier ${ }^{10}$
}

\author{
${ }^{1}$ Universität Heidelberg \\ ${ }^{2}$ Universität Trier \\ ${ }^{3}$ Universität Hildesheim \\ ${ }^{4}$ Ludwigs-Maximilian-Universität München \\ ${ }^{5}$ Universität Potsdam \\ ${ }^{6}$ Universität Mannheim \\ ${ }^{7}$ Humboldt-Universität Berlin \\ ${ }^{8}$ Technische Universität Dresden \\ ${ }^{9}$ Universität des Saarlandes, Saarbrücken \\ ${ }^{10}$ Albert-Ludwigs-Universität Freiburg
}

Die Kommission Studium und Lehre und der DGPs-Vorstand freuen sich, dass die Empfehlungen zur Qualitätssicherung in Studium und Lehre (Spinath et al., 2018) eine wesentliche Inspirationsquelle für den vorliegenden Beitrag dargestellt haben. Tatsächlich werden hochschulische Prüfungen bislang in Deutschland nur selten einer systematischen Qualitätskontrolle unterzogen, was erstaunlich ist, in Anbetracht der wichtigen Funktionen, die ihnen zukommen. Ein wesentlicher Grund dafür ist die grundgesetzlich garantierte Freiheit von Forschung und Lehre an Hochschulen. Anders als an Schulen sind die in einem Studium zu vermittelnden Inhalte weniger bis gar nicht festgeschrieben, so dass es keine inhaltlichen Standards für die verschiedenen Fächer gibt, an denen die Qualität der Vermittlung festgemacht werden könnte. Auch in der Wahl der Prüfungsformate und deren Durchführung sind die Hochschullehrenden - innerhalb gewisser gesetzlicher und institutioneller Rahmenbindungen frei und sollen es nach unserer Meinung auch bleiben. Wir sind jedoch gleichzeitig der Meinung, dass es möglich ist, qualitätssichernde Maßnahmen für hochschulische Prüfungen zu etablieren, ohne damit in die Inhalte oder Vermittlungsformen einzugreifen. Dies ist gegeben, wenn ausschließlich die messtheoretische Güte der Prüfungsformate sowie deren Rahmenbedingungen Gegenstand des Qualitätsmonitoring sind, nicht aber Prüfungsinhalte oder Vermittlungsformate. So wie die Standards zum Umgang mit Forschungsdaten (Gollwitzer et al., 2021) nicht in die Inhalte der Forschung eingreifen, wohl aber deren Qualität verbessern, so kann ein Eingriff in die Freiheit der Lehre vermieden werden, indem Standards zur Qualitätssicherung von Prüfungen primär die psychometrische Güte und Rahmenbedingungen thematisieren.

Eine dezidiert andere Auffassung als die Autorinnen und Autoren des kommentierten Beitrags haben wir in Bezug auf eine zentrale Institution, die die Qualitätssicherung hochschulischer Prüfungen übernimmt. Das als Beispiel genannte Institut für Medizinische und Pharmazeutische Prüfungsfragen (IMPP) sichert genau nicht die Qualität hochschulischer Prüfungen im Allgemeinen, sondern führt Prüfung für Staatsexamina und regulierte Studiengänge durch. Da diese Prüfungen Rückwirkungen auf die hochschulische Lehre haben, ist das IMPP verständlicherweise stetiger Kritik ausgesetzt. Zugespitzt kann man formulieren, dass das IMPP im staatlichen Auftrag Aufgaben wahrnehmen muss, die der Freiheit der Lehre an Hochschulen entgegenstehen. Es sollte tunlichst vermieden werden, weitere Institutionen dieser Art ins Leben zu rufen.

Stattdessen halten wir es für die Qualitätssicherung hochschulischer Prüfungen für unerlässlich, dass die Dozierenden selber hierfür Verantwortung übernehmen und die Hochschulen eine Infrastruktur zur Verfügung stellen, die die Lehrenden bei dieser Aufgabe unterstützt. Die Lehrenden sollten durch Angebote der Hochschuldidaktik in die Lage versetzt werden, inhaltsvalide Prüfungen zu konstruieren und standardisiert durchzuführen. An einigen Hochschulen (z.B. in den Niederlanden) wird die 
psychometrische Güte von schriftlichen Prüfungsaufgaben regelmäßig überprüft und an die Dozierenden zurückgemeldet. Diese und weitere Maßnahmen sollten an allen Hochschulen Standard werden. Gleichzeitig ist ein kollegialer und institutioneller Austausch, wie in dem Beitrag angesprochen, sehr wünschenswert. In Deutschland gibt es bislang zu wenig Angebot und Nutzung von Open Educational Resources. Zur Konstruktion guter Prüfungen wären entsprechende Repositorien, die zu einer Arbeitserleichterung jedes Einzelnen beitragen, sehr wünschenswert. Auch die Psychologie sollte eine Fachdidaktik etablieren, mit deren Hilfe Lehrende die Verantwortung für die Qualitätssicherung von Studium und Lehre inklusive dem sehr wichtigen Element der Prüfungen selbst übernehmen (vgl. Spinath et al., 2018).

\section{Literatur}

Gollwitzer, M., Abele-Brehm, A., Fiebach, C., Ramthun, R., Scheel, A. M., Schönbrodt, F. D. et al. (2021). Management und Bereitstellung von Forschungsdaten in der Psychologie: Überarbeitung der DGPs-Empfehlungen. Psychologische Rundschau, 72, 132 - 146. https://doi.org/10.1026/0033-3042/a000514

Spinath, B., Antoni, C., Bühner, M., Elsner, B., Erdfelder, E., Fydrich, T., et al. (2018). Empfehlungen der DGPs-Kommission "Studium und Lehre" zur Qualitätssicherung in Studium und Lehre. Psychologische Rundschau, 69, 183-192. https://doi. org/10.1026/0033-3042/a000408

\section{Prof. Dr. Birgit Spinath}

Pädagogische Psychologie, Psychologisches Institut

Universität Heidelberg

Hauptstr. $47-51$

69117 Heidelberg

birgit.spinath@psychologie.uni-heidelberg.de

https://doi.org/10.1026/0033-3042/a000531 